\title{
Isolated aneurysmal disease as an underestimated finding in individuals with JAG1 pathogenic variants.
}

\author{
Jotte Rodrigues Bento ${ }^{1}$, Alice Krebsová ${ }^{2}$, Ilse Van Gucht ${ }^{1}$, Irene Valdivia Callejon ${ }^{1}$, An \\ Van Berendoncks ${ }^{3}$, Pavel Votypka ${ }^{4}$, Ilse Luyckx ${ }^{1}$, Petra Peldova ${ }^{4}$, Steven Laga ${ }^{3}$, Marek \\ Havelka $^{4}$, Lut Van Laer ${ }^{1}$, Pavel Trunecka ${ }^{2}$, Nele Boeckx ${ }^{1}$, Aline Verstraeten ${ }^{1}$, Milan \\ Macek $^{4}$, Josephina Meester ${ }^{1}$, and Bart Loeys ${ }^{1}$ \\ ${ }^{1}$ Universitair Ziekenhuis Antwerpen Centrum Medische Genetica \\ ${ }^{2}$ Institut klinicke a experimentalni mediciny \\ ${ }^{3}$ Universitair Ziekenhuis Antwerpen \\ ${ }^{4}$ Fakultni nemocnice v Motole
}

February 2, 2022

\begin{abstract}
Pathogenic variants in JAG1 are known to cause Alagille syndrome (ALGS), a disorder that primarily affects the liver, lung, kidney and skeleton. Whereas cardiac symptoms are also frequently observed in ALGS, thoracic aortic aneurysms have only been reported sporadically in post-mortem autopsies. We here report two families with segregating JAG1 variants that present with isolated aneurysmal disease, as well as the first histological evaluation of aortic aneurysm tissue of a JAG1 variant carrier. Our observations shed more light on the pathomechanisms behind aneurysm formation in JAG1 variant carriers and underline the importance of cardiovascular imaging in the clinical follow-up of JAG1 variant carrying individuals.
\end{abstract}

Isolated aneurysmal disease as an underestimated finding in individuals with $J A G 1$ pathogenic variants.

Jotte Rodrigues Bento ${ }^{1}$, Alice Krebsová ${ }^{2}$, Ilse Van Gucht ${ }^{1}$, Irene Valdivia Callejon ${ }^{1}$, An Van Berendoncks ${ }^{3}$, Pavel Votypka ${ }^{4}$, Ilse Luyckx ${ }^{1,5}$, Petra Peldova ${ }^{4}$, Steven Laga ${ }^{6}$, Marek Havelka ${ }^{4}$, Lut Van Laer ${ }^{1}$, Pavel Trunecka $^{7}$, Nele Boeckx ${ }^{1}$, Aline Verstraeten ${ }^{1}$, Milan Macek ${ }^{4}$, Josephina A.N. Meester ${ }^{1}$, Bart Loeys ${ }^{1,5}$

${ }^{1}$ Centre of Medical Genetics, Antwerp University Hospital/University of Antwerp, Antwerp, Belgium

${ }^{2}$ Department of Cardiology, Center for Inherited Cardiovascular Disorders, Institute for Clinical and Experimental Medicine (IKEM), Praha 4, 14021 Prague, Czech Republic

${ }^{3}$ Department of Cardiology, Antwerp University Hospital/University of Antwerp, Antwerp, Belgium

${ }^{4}$ Department of Biology and Medical Genetics, Second Faculty of Medicine, Charles University and Motol University Hospital, Praha 5, 15006 Prague, Czech Republic

${ }^{5}$ Department of Human Genetics, Radboud University Medical Center, Nijmegen, The Netherlands

${ }^{6}$ Department of cardiac surgery, Antwerp University Hospital/University of Antwerp, Antwerp, Belgium

${ }^{7}$ Department of Hepatology and Gastroenterology, Transplant Center of Institute for Clinical and Experimental Medicine (IKEM), Praha 4, 14021 Prague, Czech Republic 
Abstract: Pathogenic variants in JAG1 are known to cause Alagille syndrome (ALGS), a disorder that primarily affects the liver, lung, kidney and skeleton. Whereas cardiac symptoms are also frequently observed in ALGS, thoracic aortic aneurysms have only been reported sporadically in post-mortem autopsies. We here report two families with segregating $J A G 1$ variants that present with isolated aneurysmal disease, as well as the first histological evaluation of aortic aneurysm tissue of a JAG1 variant carrier. Our observations shed more light on the pathomechanisms behind aneurysm formation in JAG1 variant carriers and underline the importance of cardiovascular imaging in the clinical follow-up of JAG1 variant carrying individuals.

Keywords: Alagille syndrome, JAG1, thoracic aortic aneurysm, intracranial aneurysm

Main text: JAG1 serves as one of five Notch interacting surface ligands and is ubiquitously expressed in the embryo, while expression in adult tissue is restricted to the heart, placenta, pancreas, prostate and large arteries (Carithers \& Moore, 2015; Grochowski, Loomes, \& Spinner, 2016). Given that the Notch pathway is a highly conserved signaling cascade engaged in normal development of fetal organs and in cell fate decisions in postnatal life, it is not unexpected that variants in Notch pathway components can cause various diseases (Grochowski et al., 2016). Alagille syndrome (ALGS) is one such rare heritable disorder that is predominantly caused by loss-of-function variants in JAG1 (in $94.3 \%$ of ALGS patients, another $2.5 \%$ has NOTCH2 variants, $3.2 \%$ of patients remains) and has an estimated incidence of 1:30,000 live births (Gilbert et al., 2019). ALGS is clinically diagnosed when three out of seven typical clinical features are observed, but due to marked variability (even within families), patients can remain undiagnosed. A molecular diagnosis is therefore the key to adequate patient management (Saleh, Kamath, \& Chitayat, 2016). Hepatic abnormalities, with intrahepatic duct deficiency leading to cholestasis as the most prevalent manifestation, are observed in up to $100 \%$ of patients; posterior embryotoxon has a prevalence of approximately 80-90\%; and cardiac structural changes such as Tetralogy of Fallot and peripheral pulmonary artery stenosis are present in over $90 \%$ of patients (Saleh et al., 2016; Spinner, Gilbert, Loomes, \& Krantz, 1993). Renal disease, distinct facial features, and butterfly and hemi-vertebrae are also reported frequently. Furthermore, non-cardiac vascular complications were noted to be a significant part of the clinical spectrum (estimated in $34 \%$ of ALGS patients), with intracranial aneurysm and hemorrhage as the most frequent finding (Kamath et al., 2004; Saleh et al., 2016; Spinner et al., 1993).

Using whole exome sequencing on the DNA of a fetus presenting with left hypoplastic heart syndrome and left renal agenesis (Figure 1A IV:4), a missense variant in JAG1 (NM_000214:c.2242T>C, p.Cys748Arg, ClinVar accession VCV001172525.1) was discovered. The variant (i) replaces a highly conserved and critical cysteine residue that is part of a disulfide bridge in one of the 14 EGF repeats, which are important for Notch receptor binding; (ii) is absent from control databases (including GnomAD); (iii) has not been previously reported; and (iv) was the only relevant pathogenic variant identified in this patient (Ashkenazy et al., 2016; Grochowski et al., 2016; Karczewski et al., 2020). The JAG1 variant found in the fetus was inherited from the mother (Figure 1A III:6, proband, 35 years old), who underwent surgery at the age of 10 for aortic coarctation, a feature that has been linked to ALGS before (Kamath et al., 2004). Examination of the mother and her siblings revealed absence of typical ALGS manifestations. However, in both the monozygotic twin sister (Figure 1A III:8) and brother (36 years old, Figure 1A III:11), thoracic aortic aneurysms (TAA) were discovered with a diameter of $40 \mathrm{~mm}$ (Z-score of 3.6) and $58 \mathrm{~mm}$ (Z-score of 8.6) respectively, urging the brother to undergo immediate Bentall surgery with mechanical aortic valve replacement. Histological examination of aorta obtained during surgery showed fragmentation of elastic fibers with marked decrease in elastin content (Figure 2A and 2B). Collagen was drastically increased and disorganized compared to a healthy control sample (Figure 2C and 2D). Since the histological phenotype in our patient resembles that in Marfan syndrome and Loeys-Dietz syndrome patients (Maleszewski, Miller, Lu, Dietz, \& Halushka, 2009) and dysregulated TGF $\beta$ signaling is a key feature of these syndromes, we also studied whether phosphorylated SMAD2 (pSMAD2), a downstream marker of TGF $\beta$ activity, is increased here too. Indeed, we qualitatively observed that more nuclei were positive for pSMAD2 (Figure 2F) compared to a control sample (Figure 2E). The father of the proband (Figure 1A II:7, aged 69) carried the variant and did not show any features besides aortic wall calcifications. Segregation analysis revealed presence of the variant in three siblings (II:2, II:5 and II:6; Figure 1A). Of note, the uncle of the proband (II:2 63 years old) suffered from multiple intracranial 
aneurysms (IA) and subarachnoid bleeding from ruptured arteria communicans anterior aneurysm. The aunt (II:5, aged 65) presented with bicuspid aortic valve but normal aortic measurements (sinus $34 \mathrm{~mm}$, aorta ascendens $34 \mathrm{~mm}$ ). The second aunt (II:6; aged 60) had normal echocardiographic evaluation. This marked inter-familial phenotypic variability reflects the significant and repeatedly described range in disease expression of ALGS (Gilbert et al., 2019; Guegan, Stals, Day, Turnpenny, \& Ellard, 2012). Individual IV:6 had a normal echocardiographic evaluation, but she is only 3 years old. Intriguingly, apart from the cardiac and renal phenotype of the fetus (Figure 1A IV:4), none of the patients showed any of the typical ALGS features.

In a second family, sequencing of a custom-made cardiac/aortic conditions-related gene panel revealed deletion-insertion variant of $J A G 1$ in the 42-year-old male TAA proband (Figure 1B, II:1). The variant affects the splice acceptor site and first nucleotides of exon 17(NM_000214.2: c.2114-5_2119delins18). The variant was not reported before and RNA analysis of the proband revealed in-frame skipping of exon 17 (Figure S1). No other relevant genetic variants were identified. At age 24 years, the patient underwent Bentall surgery with mechanical aortic valve replacement because of an accidental finding of aortic aneurysm (at age 22, ascending aorta $60 \mathrm{~mm}, \mathrm{Z}$-score of 10). Other family members, including the proband's sister who also carried the deletion-insertion variant, also presented with severe vascular disease. The mother of the proband (Figure 1B, I:2) underwent preventive surgery with aortic replacement for TAA at the age of 61 and died at the age of 63 of an intracranial bleeding associated with IA. The proband's sister (Figure 1B, II:2) suffered multiple IAs (left basilary and cerebral artery, arteria cerebri posterior, bilateral arteria cerebri media) and subarachnoid bleeding events, but showed normal aortic dimensions. The proband's daughter (Figure 1B, III:2 - age 13 years), which carries the mutation, had a normal total body MR angiography. In accordance with the first family, patients in family B (Figure 1B, II:2, II:1, II:2) did not present with typical ALGS hepatic, ocular or skeletal characteristics.

We are the first to report two ALGS families with isolated aneurysmal disease lacking other ALGS pathognomonic clinical characteristics (such as the hepatic abnormalities, posterior embryotoxon and cardiac structural changes described above). In the current ALGS literature there is no notion of TAA in the vast majority of ALGS patient descriptions, but systematic aortic evaluation might not be performed on a routine basis. TAA has been reported before in only four ALGS patients, three of which were discovered at autopsy (Kamath et al., 2004; Molinero-Herguedas, Labrador-Fuster, Rios-Lazaro, \& Carmaniu-Tobal, 2008). In all four, at least one typical clinical ALGS manifestation was present. In the patient reported by Molinero-Herguedas et al., TAA was discovered during reexamination of an (undefined) cardiopathy and three patients reported in Kamath et al. had characteristic facial features, but due to discovery at autopsy no details on other organ system involvement were available. Similar for IA in ALGS, which has an estimated prevalence of $14 \%$, patients previously reported always presented accompanying cholestatic liver disease and cardiac defects (Kamath et al., 2004).

For the first time, histological evaluation of aortic tissue of a TAA patient with a JAG1 variant was performed, revealing an unexpected phenotype with elastin degradation and abnormal collagen deposition. Fibrosis has been associated with JAG1 gain-of-function rather than loss-of-function in fibrotic kidney disease, where excessive Notch signaling is known to induce disproportionate expression of extracellular matrix (ECM) components (Hu et al., 2015). Additionally, in a mouse model of abdominal aortic aneurysm, pharmacological inhibition of Notch signaling resulted in regression of aneurysm along with a reduction in elastic fiber fragmentation and collagen deposition (Sharma et al., 2019). On the other hand, it has been observed that dysfunction of Notch signaling mitigates epithelial-to-mesenchymal transition (EMT), an indispensable process during cardiovascular development and repair mechanisms. Additionally, contractile markers of vascular smooth muscle cells (VSMC) are downregulated upon Notch impairment, suggesting that the VSMC in the aortic media of ALGS patients adopt a synthetic phenotype, characterized by increased ECM synthesis and elastolysis due to secretion of matrix metalloproteases. Resulting tissue damage and subsequent failure to sufficiently dampen hemodynamical pressure could trigger TGF $\beta$ signaling, which is expressed by increased pSMAD2 in our patient. Knowing that TGF $\beta$ is a well-known mediator of fibrosis and a driver of metalloprotease activity, a destructive cycle is initiated, leading to aortic wall degradation (Jones, Spinale, \& 
Ikonomidis, 2009; Kostina et al., 2016; Zavadil, Cermak, Soto-Nieves, \& Bottinger, 2004). Further functional studies will be necessary to determine the true sequence of events of ALGS related TAAD.

With the current report of these patients with significant Z-scores and intracranial hemorrhaging at a relatively young age without obvious ALGS findings, we want to urge clinicians to include JAG1 (andNOTCH2 ) in genetic screening panels for TAA and IA as this is not yet the case in most clinics - most probably due to insufficient clinical evidence (Renard et al., 2018), which we now provide. Furthermore, we anticipate TAA might be an underestimated finding in ALGS and we therefore advise to early and thoroughly examine the vascular system in such patients, with focus on intracranial and thoracic aorta imaging, since vascular events are estimated to account for over 30\% of ALGS mortality (Kamath et al., 2004). A systematic large-scale ALGS cohort investigation could straighten out the true significance of aortopathy in ALGS.

\section{Materials and methods}

\section{Whole exome sequencing:}

DNA of the fetus and parents (Figure 1A IV:4) was subjected to whole exome sequencing (WES) according to the manufacturer's protocols. DNA was fragmented using the Covaris M220 Focused-ultrasonicator and subsequently prepped with the TruSeq DNA Sample Preparation kit (Illumina). The SeqCap EZ Human Exome Library v3.0 kit was used for enrichment (NimbleGen, Roche) and finally, sequencing was carried out on a HiSeq 2000 (Illumina). Analysis of WES data was performed using VariantDB (Vandeweyer, Van Laer, Loeys, Van den Bulcke, \& Kooy, 2014). Sanger sequencing was performed to confirm the presence of the variant in the fetus and subsequently to perform segregation analysis in the other family members. A PCR reaction using GOTaq polymerase (Promega) was followed by sequencing using the ABI BigDye Terminator V3.1 Cycle Sequencing kit (Applied Biosystems) and the ABI 3130 Genetic Analyzer (Applied Biosystems).

DNA of members of family B (II:1, II:2 and III:1-4) was subjected to a custom-made panel comprising 100 cardiac/aortic conditions-related candidate genes (SOPHiA Genetics, Switzerland) according to the manufacturer's protocols. The sequencing was carried out on a MiSeq (Illumina). Variant prioritization was performed by Sophia DDM software (Sophia Genetics, Switzerland). The evaluation was carried out by bioinformatics tools integrated in Varsome Clinical software (Saphetor SA, Switzerland). Presence of the detected variant was validated by Sanger DNA sequencing and family segregation was performed.

\section{Splicing analysis of family B}

RNA analysis was performed from blood of the proband drawn into a PAXgene Blood RNA Tube, followed by RNA isolation and a reverse transcriptase reaction followed by Sanger sequencing covering JAG1 exons 16-18 (primers: F: GGCCTACTGTGAAACCAATATT, R:TCATTGGTATTCTGAGCACAG).

\section{Elastic fiber, collagen and pSMAD2 staining}

Collected aortic tissue was embedded in paraffin and cut into $5 \mu \mathrm{m}$ thick sections. Elastic fiber integrity and collagen content were assessed using Verhoeff Van Gieson (Sigma-Aldrich) and Trichrome Masson's (SigmaAldrich), respectively, according to manufacturer's instructions. pSMAD2 was visualized using immunohistochemistry. Deparaffinization in toluene ( 5 minutes) was followed by rehydration in $100 \%, 90 \%, 70 \%$ and $50 \%$ ethanol solutions, after which sections were subjected to $3 \%$ hydrogen peroxide followed by incubation in trypsin for 10 minutes at $37^{\circ} \mathrm{C}$ (Sigma-Aldrich, 93615-25G). Sections were heated in citrate buffer for 10 minutes at $90^{\circ} \mathrm{C}$ and blocked for 20 minutes at room temperature (RT) with goat serum (Vector-lab consult). A primary pSMAD2 antibody (3101, Cell Signaling; 1:5000) was incubated overnight using a concentration of 1:5000 and the following day, the sections were incubated with the secondary antibody (30014 secondary goat anti-rabbit IgG, Vector; 1:200) for $30 \mathrm{~min}$ and subsequently with an avidin-biotinylated complex for 1 hour, both at RT (Vectastain ABC kit, Vector Laboratories). 3,3-Diaminobenzidine tetrahydrochloride hydrate (DAB) chromogen (Sigma-Aldrich) was used as the substrate and Hematoxylin as counterstaining. All pictures were acquired using a Leica DMi8 inverted microscope, at a 20x magnification.

Figure 1: Pedigrees of two families with JAG1 variants. A) family with p.Cys748Arg variant; B) family 
with the c.2114-5_2119delins18 variant.

Figure 2: Histological staining of elastin (A and B), collagen (C and D), and phosphorylated SMAD2 protein (pSMAD2, brown nuclear staining, $\mathrm{E}$ and $\mathrm{F}$ ) of aortic tissue of a healthy control (top) and patient A:III:11 (bottom). Scalebar: $100 \mu \mathrm{m}$.

Figure S1: Splicing analysis of the insertion-deletion variant encompassing the splice acceptor site and first nucleotides of exon 17 (NM_000214.2: c.2114-5_2119delins18).

\section{Acknowledgments}

This research was largely supported by funding from the University of Antwerp (GOA 33933; MethusalemOEC grant "Genomed" 40709), the Research Foundation Flanders (FWO, Belgium, G042321N, G040221N, G044720N), the Dutch Heart Foundation (2013T093), the Belgian Cardiac Surgery Foundation and the Marfan Foundation. Dr. Loeys holds a consolidator grant from the European Research Council (Genomia - ERC-COG-2017-771945). Dr Luyckx is supported by the Dutch Heart Foundation (Outreach project). Milan Macek and Alice Krebsova get support from Czech Medical Research Council project n. NV 18-0200237. Josephina Meester and Ilse Van Gucht are fellows of the Fund for Scientific Research Flanders. Jotte Rodrigues Bento is supported by De Swerts Fund.

\section{Data availability statement}

Additional data on the RNA splicing experiment is available in the article supplementary material.

\section{Conflict of interest}

The authors declare no conflict of interest.

\section{References}

Ashkenazy, H., Abadi, S., Martz, E., Chay, O., Mayrose, I., Pupko, T., \& Ben-Tal, N. (2016). ConSurf 2016: an improved methodology to estimate and visualize evolutionary conservation in macromolecules. Nucleic Acids Res, 44 (W1), W344-350. doi:10.1093/nar/gkw408

Carithers, L. J., \& Moore, H. M. (2015). The Genotype-Tissue Expression (GTEx) Project. Biopreserv Biobank, 13 (5), 307-308. doi:10.1089/bio.2015.29031.hmm

Gilbert, M. A., Bauer, R. C., Rajagopalan, R., Grochowski, C. M., Chao, G., McEldrew, D., . . . Spinner, N. B. (2019). Alagille syndrome mutation update: Comprehensive overview of JAG1 and NOTCH2 mutation frequencies and insight into missense variant classification. Hum Mutat, 40 (12), 2197-2220. doi:10.1002/humu.23879

Grochowski, C. M., Loomes, K. M., \& Spinner, N. B. (2016). Jagged1 (JAG1): Structure, expression, and disease associations. Gene, 576 (1 Pt 3), 381-384. doi:10.1016/j.gene.2015.10.065

Guegan, K., Stals, K., Day, M., Turnpenny, P., \& Ellard, S. (2012). JAG1 mutations are found in approximately one third of patients presenting with only one or two clinical features of Alagille syndrome.Clin Genet, 82 (1), 33-40. doi:10.1111/j.1399-0004.2011.01749.x

Hu, C., Sun, L., Xiao, L., Han, Y., Fu, X., Xiong, X., . . . Kanwar, Y. S. (2015). Insights into the Mechanisms Involved in the Expression and Regulation of Extracellular Matrix Proteins in Diabetic Nephropathy. Curr Med Chem, 22 (24), 2858-2870. doi:10.2174/0929867322666150625095407

Jones, J. A., Spinale, F. G., \& Ikonomidis, J. S. (2009). Transforming growth factor-beta signaling in thoracic aortic aneurysm development: a paradox in pathogenesis. J Vasc Res, 46 (2), 119-137. doi: $10.1159 / 000151766$

Kamath, B. M., Spinner, N. B., Emerick, K. M., Chudley, A. E., Booth, C., Piccoli, D. A., \& Krantz, I. D. (2004). Vascular anomalies in Alagille syndrome: a significant cause of morbidity and mortality.Circulation, 109 (11), 1354-1358. doi:10.1161/01.CIR.0000121361.01862.A4 
Karczewski, K. J., Francioli, L. C., Tiao, G., Cummings, B. B., Alfoldi, J., Wang, Q., . . . MacArthur, D. G. (2020). The mutational constraint spectrum quantified from variation in 141,456 humans. Nature, 581 (7809), 434-443. doi:10.1038/s41586-020-2308-7

Kostina, A. S., Uspensky Vcapital Ie, C., Irtyuga, O. B., Ignatieva, E. V., Freylikhman, O., Gavriliuk, N. D., . . . Malashicheva, A. B. (2016). Notch-dependent EMT is attenuated in patients with aortic aneurysm and bicuspid aortic valve. Biochim Biophys Acta, 1862 (4), 733-740. doi:10.1016/j.bbadis.2016.02.006

Molinero-Herguedas, E., Labrador-Fuster, T., Rios-Lazaro, M., \& Carmaniu-Tobal, J. (2008). [Aortic aneurysm in Alagille syndrome].Rev Esp Cardiol, 61 (6), 658-659. Retrieved from https://www.ncbi.nlm.nih.gov/pubmed/18570795

Renard, M., Francis, C., Ghosh, R., Scott, A. F., Witmer, P. D., Ades, L. C., . . . De Backer, J. (2018). Clinical Validity of Genes for Heritable Thoracic Aortic Aneurysm and Dissection. J Am Coll Cardiol, 72 (6), 605-615. doi:10.1016/j.jacc.2018.04.089

Saleh, M., Kamath, B. M., \& Chitayat, D. (2016). Alagille syndrome: clinical perspectives. Appl Clin Genet, 9, 75-82. doi:10.2147/TACG.S86420

Sharma, N., Dev, R., Ruiz-Rosado, J. D., Partida-Sanchez, S., Guerau-de-Arellano, M., Dhakal, P., . . . Hans, C. P. (2019). Pharmacological inhibition of Notch signaling regresses pre-established abdominal aortic aneurysm. Sci Rep, 9 (1), 13458. doi:10.1038/s41598-019-49682-0

Spinner, N. B., Gilbert, M. A., Loomes, K. M., \& Krantz, I. D. (1993). Alagille Syndrome. In M. P. Adam, H. H. Ardinger, R. A. Pagon, S. E. Wallace, L. J. H. Bean, G. Mirzaa, \& A. Amemiya (Eds.), GeneReviews((R)) . Seattle (WA).

Vandeweyer, G., Van Laer, L., Loeys, B., Van den Bulcke, T., \& Kooy, R. F. (2014). VariantDB: a flexible annotation and filtering portal for next generation sequencing data. Genome Med, 6 (10), 74 . doi:10.1186/s13073-014-0074-6

Zavadil, J., Cermak, L., Soto-Nieves, N., \& Bottinger, E. P. (2004). Integration of TGF-beta/Smad and Jagged1/Notch signalling in epithelial-to-mesenchymal transition. EMBO J, 23 (5), 1155-1165. doi:10.1038/sj.emboj.7600069

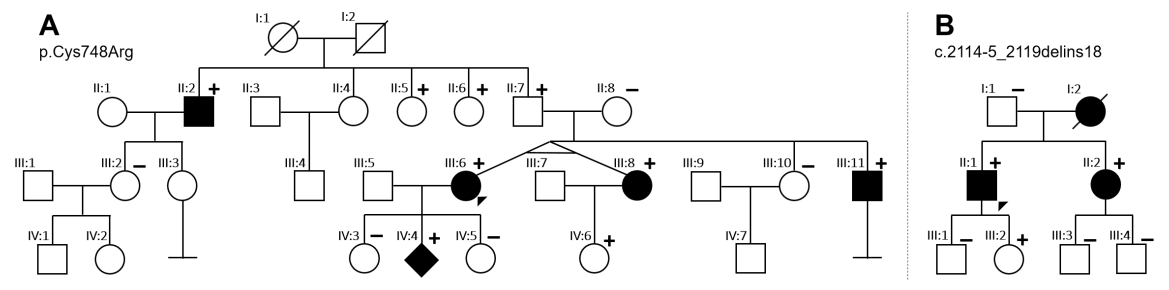



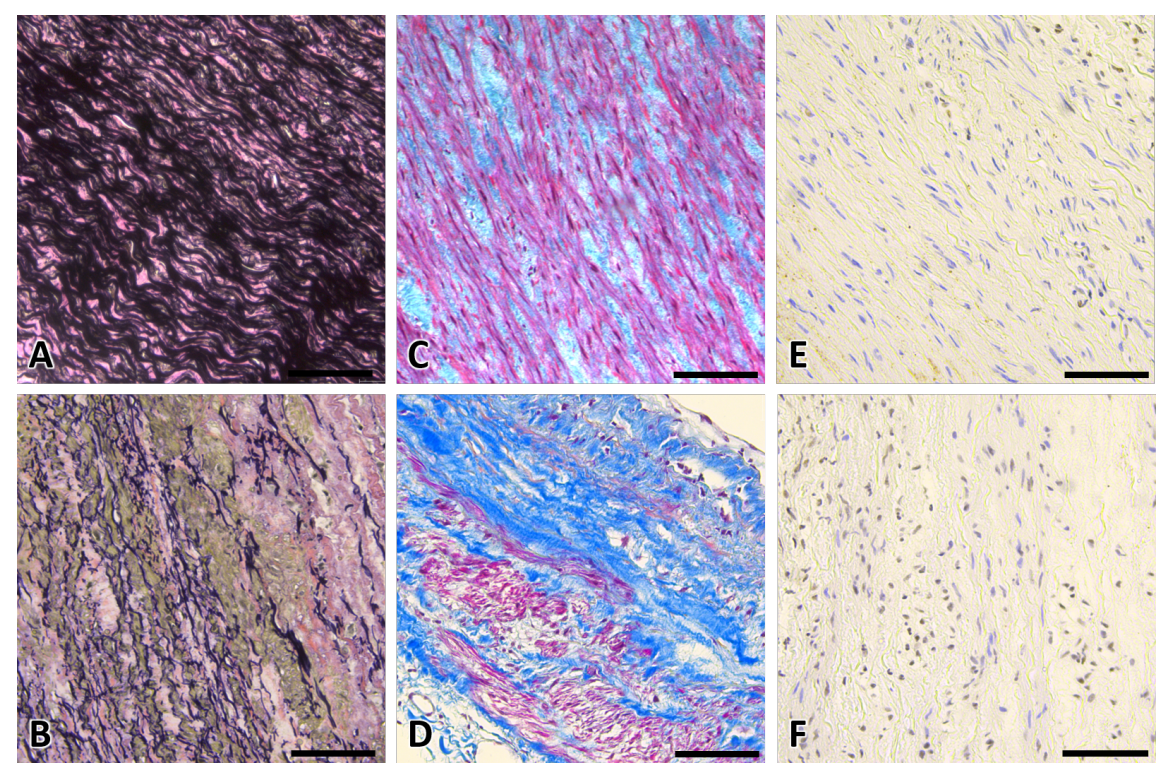